\title{
Estratégia Competitiva e Estratégia de Produção: o Caso de uma Empresa de Cosméticos
}

\author{
Rosangela Maria Vanalle \\ UNIMEP. Rodovia Sta Bárbara - Iracemápolis, Km 1. CEP 13450-000 Santa Bárbara do Oeste SP. \\ E-mail:rvanalle@zaz.com.br
}

Alceu Gomes Alves Filho

UFSCar. Via Washington Luiz, Km 235. CEP 13565-905 São Carlos SP.

E-mail: alcen@power.ufscar.br

Marilia Gabriela Pavan Kuri

UFSCar - Rodovia Washington Luiz, Km 235, São Carlos - SP

grupok@zaz.com.br_fabercos@linkway.com.br

\section{Resumo}

Este artigo identifica e discute a estratégia de produção (EP) adotada em uma empresa de cosméticos. Esta investigação considera questões relacionadas ao conteúdo e ao processo de formulação da EP e foi conduzida por um time composto por dois pesquisadores e três profissionais vinculados à empresa. Nesse processo de pesquisa-ação, a equipe concluiu que a EP deveria ser modificada principalmente no que diz respeito aos períodos de implantação dos programas nas áreas estruturais e infra-estruturais, ao grau de formalização do processo de formulação e à abrangência do sistema de avaliação de desempenho

\begin{abstract}
This paper identifies and discusses the manufacturing strategy (MS) adopted in a cosmetics firm. This investigation considered issues related to the content and process of formulating MS and was carried out by a team of two researchers and three professionals of the firm. In this "action research" process, the team concluded that the MS required modifications, mainly in regard to the timing of implementation of programmes in the structural and infrastructural decision areas, the degree of formalization of the strategy formulation process, and scope of the performance evaluation system.
\end{abstract}

Keywords: manufacturing strategy, competitive strategy, cosmetics manufacturer.

\section{Introdução}

Este trabalho tem como objetivo relatar um estudo sobre a Estratégia de Produção (EP) de uma unidade de negócios, a "Divisão de Cosméticos" (DC) de uma empresa brasileira. Por tratar-se de um estudo exploratório, com muitas variáveis intervenientes, optou-se por uma análise qualitativa, abrangendo as etapas de identificação, discussão e reelaboração da estratégia de produção da unidade.

O interesse pelo assunto, sua relevância dos pontos de vista acadêmico e prático e a escassa literatura sobre estudos empíricos contemplando concomitantemente conteúdo e processo da EP (MILLS et al, 1995 e LEONG et al, 1990) motivaram a realização de tal estudo. Procurou-se desenvolvê-lo em uma empresa em que se pudesse contar com informações fidedignas sobre tais assuntos e, também, em que se pudesse participar efetivamente do processo de identificação e discussão da EP.

A Unidade de Negócios Cosméticos foi estruturada recentemente na empresa estudada, tornando-se uma Divisão da Companhia semi-autônoma. Com essa nova estruturação houve a necessidade de se elaborar um diagnóstico da Estratégia Competitiva da Unidade, a fim de se determinar se tal estratégia coaduna-se com a missão e objetivos maiores da Divisão. Surgiu também a necessidade de rever, alterar ou 
mesmo redefinir as estratégias funcionais. Neste artigo, a estratégia funcional a ser focalizada será a Estratégia de Produção. O estudo dessa estratégia engloba a identificação das prioridades competitivas da produção dessa Unidade para o período 2000-2001, bem como a definição de um vínculo claro e formal com a Estratégia Competitiva da Divisão.

Todos esses pontos são de fundamental importância, principalmente se for considerado o período crítico recentemente vivenciado pela empresa - que procura dar novo direcionamento estratégico ao negócio - porque dá as bases para um estudo aprofundado, por parte da Gerência, sobre quais investimentos priorizar para atingir os objetivos desejados de vendas e participação no mercado doméstico e internacional.

O estudo de caso nessa empresa, que passa pela reformulação de suas estratégias, pode auxiliar no esclarecimento de algumas das questões ainda não suficientemente discutidas na literatura sobre Estratégias Competitiva e de Produção (porque há pouco respaldo empírico para a discussão de pontos importantes relacionados ao assunto), fornecendo uma ilustração de um processo de planejamento estratégico da produção.

Para as fases de identificação e discussão da estratégia de produção atual, utilizou-se o seguinte método:

Discutiram-se um roteiro de entrevistas que já vinha sendo utilizado em outras pesquisas para identificação dos conteúdos e processos relacionados às estratégias competitivas e de produção em empresas. Foram feitas, então, algumas adaptações nesse roteiro, seguindo especialmente as sugestões dos administradores da empresa.

Realizaram-se entrevistas com dois dos gerentes da Divisão: com o primeiro, gerente geral da divisão e da área de marketing, abordaram-se questões relacionadas às estratégias competitivas e de marketing e, ainda, às prioridades competitivas da função produção. Com o segundo, gerente de produção, tratou-se das mesmas questões sobre prioridades competitivas abordadas com o primeiro entrevistado e também de questões relacionadas aos objetivos de produção, programas e mudanças implementadas na produção nos últimos dois anos.
Compilaram-se e discutiram-se as respostas dos dois gerentes acima, inicialmente, com uma engenheira de produção subordinada ao gerente de produção (também participante deste artigo), oportunidade em que foram esclarecidas diversas dúvidas dos dois pesquisadores sobre os assuntos tratados e sobre alguns poucos pontos de aparente conflito entre as posições e percepções dos dois gerentes mencionados.

Discutiram-se os resultados do trabalho, procurandose identificar as estratégias competitivas e de produção que vinham sendo adotadas, provocando uma reflexão sobre as recentes decisões tomadas. Os resultados organizados das entrevistas "funcionaram" como um "espelho" das estratégias até então implementadas. A seguir, foi apresentada uma avaliação das estratégias competitiva e de produção até então adotadas e traçou-se, em linhas gerais, e considerando as manifestações de ambos os gerentes quanto às mudanças que estavam para ser implementadas, o que poderia ser uma EP adequada e compatível com a estratégia competitiva a curto prazo.

Seguiu-se uma longa discussão sobre a estratégia atual e sobre alternativas de estratégias para o futuro próximo. Diversos programas de ações foram sugeridos, que seriam implementados conforme alguns resultados desejados fossem sendo alcançados e em função das condições internas e externas à empresa. Esses programas estão sendo ainda examinados para então serem selecionados e alocados (no horizonte de planejamento) como partes de planos estratégicos alternativos.

Nas seções seguintes será abordada de modo sucinto a estrutura conceitual que orienta esse tipo de investigação e será apresentado, com mais detalhes, o caso analisado com o método acima descrito.

\section{Estratégia Competitiva (EC) e Estratégia de}

\section{Produção (EP)}

As mudanças no ambiente competitivo mundial alteraram sensivelmente as concepções básicas de como produzir bens. A emergência do novo paradigma de 
produção japonês e a globalização da economia provocaram o aumento na competição mundial e concretizaram a necessidade das empresas terem de substituir antigas premissas por uma visão mais dinâmica, de longo prazo, e, principalmente, mais orientada ao cliente.

Alguns autores identificam como fundamentos estruturais da competitividade o estímulo dado às empresas por mercados internos dinâmicos e exigentes, a elevação do conteúdo tecnológico dos produtos, liderada pelas grandes empresas do setor, e a presença sistemática das empresas em mercados internacionais (COUTINHO \& FERRAZ, 1994). Para esses autores, as indústrias passaram (ou ainda passam) por uma transição de um paradigma industrial que utilizava exclusivamente as vantagens competitivas tradicionais, como disponibilidade de recursos naturais e energia, para um novo paradigma, voltado para outros elementos que garantiriam a competitividade: porte industrial e integração produtiva que permitam estratégias agressivas, fortalecimento de redes cooperativas horizontais (por exemplo, alianças estratégicas) e a intensificação da cooperação vertical para otimizar capacitações produtivas e tecnológicas nas cadeias.

Assim, as tendências apontadas de formação de cadeias, contínua busca pela inovação, ambientes cada vez mais dinâmicos, a intenção de participação das empresas cada vez maior no mercado internacional e o ambiente concorrencial direcionam as empresas a desenvolver uma visão de longo prazo, garantindo um ritmo constante de inovações e dinamismo.

Esse novo cenário mundial, com a transformação do ambiente competitivo, aumentou o nível de incertezas e tornou imprescindível para qualquer empresa o desenvolvimento de métodos, processos e tecnologias mais flexíveis para acompanhar tais mudanças. No entanto, a competitividade depende de mais do que isso: o sucesso de uma empresa requer também um planejamento estratégico adequado, reforçando a premissa de que é necessário entender o sistema de produção como um elemento estratégico, no qual alterações devem ser implementadas para se incrementar a competitividade da empresa. E surgiram, então, os primeiros estudos relacionados aos conceitos de Estratégia Competitiva (EC) e Estratégia de Produção (EP).

De modo geral, pode-se dizer que estratégia é um padrão ou plano que integra os grandes objetivos, as políticas e as seqüências de ações de uma organização em um todo coeso (QUINN, 1988). E a estratégia empresarial possui três níveis hierárquicos, segundo WHEELWRIGHT (1984): Estratégia Corporativa ou Global ( $1^{\circ}$ nível), Estratégia Competitiva ou da Unidade de Negócio (nível médio) e Estratégia Funcional ( $3^{\circ}$ nível). Dentre as estratégias funcionais estaria a Estratégia de Produção (EP), ao lado de outras como Estratégia de Marketing, Estratégia Financeira e Estratégia Tecnológica.

Resumidamente, são apresentados aqui os conceitos principais que norteiam a elaboração desta pesquisa sobre a EP em uma empresa. Uma revisão mais aprofundada pode ser encontrada em VANALLE (1995).Tratando-se de uma estratégia funcional da empresa, subordinada a sua estratégia competitiva, faz-se necessário também, ainda que de modo superficial, certa compreensão do conceito de estratégia competitiva para a elaboração deste trabalho.

\subsection{Estratégia Competitiva (EC)}

Estratégia competitiva (EC) é o conjunto de planos, políticas, programas e ações desenvolvidos por uma empresa ou unidade de negócios com o intuito de ampliar ou manter, de modo sustentável, suas vantagens competitivas frente aos concorrentes. Esta é uma definição elaborada a partir dos trabalhos de PORTER (1980), PORTER (1985), PRAHALAD \& HAMEL (1998) e OHMAE (1998).

Segundo COUTINHO \& FERRAZ (1994), o nível de competitividade alcançado pela empresa ou unidade de negócios depende de fatores sistêmicos, estruturais ou empresariais, relacionados, respectivamente, às condições macroeconômicas, político-institucionais, regulatórias, infra-estruturais e sociais do país onde a empresa está instalada; às características do mercado, da concorrência e da configuração da indústria ou setor econômico em que a 
PRODUÇÃO

empresa atua; e à capacidade gerencial e operacional da própria empresa.

Quanto aos fatores estruturais, relacionados à competitividade das empresas, PORTER (1985) indica as cinco forças que determinam a dinâmica da competição em uma indústria: a entrada de novos concorrentes, a ameaça de substitutos, o poder de barganha dos clientes, o poder de barganha dos fornecedores e a rivalidade entre os concorrentes atuais. E ainda segundo o mesmo autor (PORTER, 1980), as empresas podem adotar três tipos de estratégia competitiva: estratégia de liderança em custos, estratégia de diferenciação e estratégia de foco. A primeira visa obter vantagens competitivas pela oferta de produtos e serviços (em geral padronizados) a custos mais baixos do que os concorrentes. A segunda busca alcançar vantagens pela introdução de um ou mais elementos de diferenciação nos produtos e serviços, que justifiquem preços mais elevados. E o terceiro tipo de estratégia objetiva obter vantagens competitivas, ou pela oferta de produtos e serviços com menores custos, ou pela diferenciação dos mesmos, mas em um segmento de mercado mais localizado ou restrito.

A EC, para ser posteriormente implementada, deve ser desdobrada nas estratégias funcionais de marketing, produção, tecnologia, finanças e outras, dependendo da estrutura organizacional da empresa (e que também depende de sua estratégia). Nosso foco recai aqui sobre a EP.

\subsection{Estratégia de Produção (EP)}

O conceito original de conteúdo e de processo da EP foi elaborado por SKINNER (1969). Este autor e outros como HAYES (1985), WHEELWRIGHT (1984), HÖRTE et al (1987), LEONG et al (1990) e VANALLE(1995) caracterizam a EP como uma coleção de decisões individuais que afetam a capacidade da empresa em encontrar seus objetivos a longo prazo.

Segundo esses autores, as prioridades competitivas necessárias para competir com sucesso deveriam refletir a EC e também fornecer os critérios ou a missão que a função produção deveria buscar alcançar e pela qual seria avaliada. Essa missão é representada em geral por cinco diferentes dimensões ou prioridades competitivas da produção: custo, entrega, flexibilidade, qualidade e serviço (GARVIN, 1993).

Estas prioridades orientam as decisões, os programas e as ações implementados nas áreas de decisão estruturais (Localização e capacidade, Instalações e equipamentos, Tecnologia de produto e processo e Integração vertical) e infra-estruturais (Organização, Gestão de recursos humanos, Logística e planejamento e controle da produção e Gestão de qualidade).

Assim, a EP constitui-se dos planos, políticas e programas de ações implementados pela empresa, mais especificamente pela função produção (nas áreas de decisão), para que as prioridades competitivas da produção (de custo, qualidade, flexibilidade, entrega e serviço) sejam alcançadas, em consonância com as demais estratégias funcionais e com a estratégia competitiva da empresa.

Mas, além de referir-se ao conteúdo (“content”), a expressão "estratégia de produção" tem sido utilizada também para se referir ao processo ("process") de formulação da mesma (LEONG et al, 1990 e SWAMIDASS et al, 1987). Segundo VANALLE (1995), o conteúdo focaliza o que é decidido, enquanto o processo diz respeito a como as decisões são tomadas na empresa.

Já há algum tempo se reconhece o processo de formulação da EP como uma lacuna importante no desenvolvimento teórico da área. LEONG et al (1990), por exemplo, indicam a necessidade de se realizarem estudos empíricos que objetivem desvendar como as organizações elaboram suas EPs. MILLS et al (1995) argumentam que o aperfeiçoamento dos processos para desenvolvimento e implementação de estratégias representa ainda significativo desafio para pesquisadores e profissionais.

Pode-se afirmar, no entanto, que o processo de formulação e implementação é contingente do modelo de “conteúdo" da EP adotado (MILLS et al, 1995) e, assim, seria recomendável propor um método de formulação e reformulação a partir, entre outros fatores, de uma concepção e de uma pré-definição do conteúdo da EP. De acordo com MILLS et al (1995), além da concepção de EP, 
outros fatores deveriam ser considerados: as qualidades requeridas dos resultados do processo e os contextos interno e externo da empresa. HILL (1993), PLATTS - GREGORY (1989) (apud VOSS, 1992) e SLACK (1993) propuseram métodos alternativos (mas com diversos pontos comuns) para a formulação de estratégias de produção.

Neste trabalho, serão tratados tanto o conteúdo como o processo de formulação da EP em uma unidade de negócios de um empresa, entendendo-os como bastante imbricados, mas o conteúdo será tratado de modo mais detalhado. Quanto ao processo de formulação da EP, fundamentalmente está se propondo que a empresa passe a adotar uma sistemática formal de formulação e reformulação da EP.

\section{Identificação e Discussão da Estratégia}

\section{Competitiva e Estratégia de Produção da Divisão Cosméticos.}

A Divisão Cosméticos é uma das nove divisões (unidades de negócios) de uma empresa que atua nos setores de material de desenho, escrita e pintura no ramo químico, tendo se estruturado em meados de 1998 como uma Unidade de Negócios autônoma. A empresa definiu essa Unidade como um centro de custo separado, com uma gerência própria, subordinada diretamente aos stockholders da Companhia, possuindo além de um setor de produção, um setor de Marketing e Vendas também próprio. Outras atividades estão sendo gradualmente absorvidas pela Divisão, como o planejamento e compra de matérias-primas. Ainda são utilizados da Companhia os setores de Recursos Humanos e Contabilidade e Custos.

Embora tenha uma escala de produção bem menor que as outras Divisões da Companhia, ela assumiu nos últimos anos um importante papel estratégico, pois representa um segmento de mercado para lápis muito diferente daquele em que a Companhia tradicionalmente atua. Esse nicho de mercado tem se mostrado não somente relevante, mas em franca expansão, justificando a renovação do interesse da Companhia em manter-se competitiva nesse setor.
Os produtos fabricados pela Divisão são lápis cosméticos. Há uma separação por características de processo, principalmente, em: lápis delineadores com minas extrudadas, lápis delineadores com minas moldadas, lápis, jumbos, pintura facial infantil em lápis e em pote. A diferença está basicamente no processamento da mina, mais do que no próprio lápis. A fabricação de pintura em potes é por envase, sem a utilização de madeira.

Quanto ao lápis mais comum, a essência do processo de produção é a seguinte:

- Fabricação das massas: compreende a pesagem, composição e mistura dos componentes;

- Confecção da minas: produção das minas cosméticas por extrusão ou moldagem;

- Encolamento: confecção dos sanduíches de madeira. As minas são entrepostas em duas tabuinhas de madeira pré usinadas e fixadas com cola;

- Usinagem dos lápis: transformação dos sanduíches em lápis retificados e no comprimento certo;

- Pintura: várias demãos de tinta e verniz, preparando-o para o acabamento;

- Gravação: início do acabamento. O lápis é impresso por hot-stamping, silk-screen ou dry-off-set. Normalmente, cada cliente ou item produzido possui uma única decoração;

- Acabamento do topo: o topo é arredondado e recebe demãos de tinta, formando a "gota";

- Apontamento: os lápis são apontados;

- Escolha e limpeza: os lápis são escolhidos e limpos, e aqueles com defeito são retrabalhados ou refugados;

- Colocação de protetores: os lápis são tampados;

- Controle de qualidade: inspeção segundo normas ISO 9000;

- Empacotamento e expedição.

Os produtos são feitos somente sob encomenda, pois são personalizados para cada cliente. Assim, os pedidos entram em um sistema ERP integrado e o prazo é fixado por volume solicitado. Os componentes e materiais utilizados têm suas necessidades geradas via MRP, feito pelo mesmo sistema integrado. Não há terceirização de componentes. 
No chão de fábrica, após o encolamento, o fluxo de materiais e produtos é controlado por um kanban de supermercado.

Ainda em linhas gerais, é interessante saber como a Unidade de Negócios relaciona-se estruturalmente com a Companhia, visto que a Estratégia Corporativa dá o direcionamento mais geral para suas diversas Unidades. Entender qual o papel da Divisão é fundamental para estabelecer qual será a estratégia adotada por ela.

Tradicionalmente, o negócio da Companhia não engloba a fabricação de lápis cosméticos. Apenas recentemente começou a fazer parte da Estratégia Corporativa atender esse segmento de mercado, assumindo uma posição semelhante à matriz, situada na Alemanha . Assim, tendo sido observado que o mercado brasileiro, principalmente, não possuía fornecedores para este tipo de produto, instalou-se uma seção produtiva para lápis cosméticos. Com a resposta positiva do mercado, o segmento expandiu-se e hoje tem relevância para o negócio da Companhia, como um todo.

No início, a produção de lápis cosméticos não tinha identidade própria, não havendo preocupação em desenvolver e formalizar uma Estratégia: a seção cosméticos orientava-se por outras seções da Companhia, sem levar em conta diferenças fundamentais no produto e principalmente, no mercado consumidor. A diferenciação no produto, no entanto, gerou algumas dificuldades para o setor: problemas gerais no abastecimento de matériasprimas e materiais de consumo, dificuldades de entrosamento com as outras funções como Recursos Humanos, Pesquisa \& Desenvolvimento, Marketing, etc.. A Estratégia da Companhia e das outras Divisões não contemplavam tais diferenças, dificultando inclusive o fluxo de informações entre níveis hierárquicos.

Apesar de ter sido a identidade e a autonomia da Unidade prejudicadas com a miscigenação das Estratégias, é possível apontar algumas principais vantagens que a Corporação oferece para a Unidade de Negócios Cosméticos:

- Vantagem da marca: a marca da Companhia é reconhecida mundialmente, tendo uma imagem positiva e muito forte. $\mathrm{O}$ fato dos produtos da Divisão levarem essa marca aos clientes facilita muito a conquista de mercados;

- Poder de barganha forte com fornecedores e clientes, por ser uma Empresa de grande porte;

- Barreiras à entrada: porte da empresa e posicionamento de oligopóiio no mercado de lápis em geral;

- Infra-estrutura pronta para o abastecimento de madeira (plantações e planta fabril para preparação de madeira) e de componentes plásticos injetados.

É importante ressaltar que tais vantagens só são desfrutadas pela Divisão se for do conhecimento de todos os níveis hierárquicos e também das outras áreas da empresa que a Estratégia da Divisão pode ser diferente das demais, pois somente esse consenso pode garantir que haja colaboração e sintonia entre setores.

\subsection{Identificação da Estratégia Competitiva da} Divisão Cosméticos

Em entrevista com o gerente da unidade de negócios e responsável também por sua área de marketing, foram obtidas, como já indicado, as informações consideradas relevantes sobre a estratégia competitiva da empresa.

Discutindo tais informações, a equipe concluiu que a EC poderia ser assim explicitada: Satisfazer os clientes, oferecendo ampla variedade de produtos com alta qualidade de desempenho funcional e de acabamento, a preços relativamente baixos e dentro dos prazos estabelecidos. Devem ser exploradas para isso as competências e vantagens relativas acumuladas pela empresa especialmente no mercado interno tais como acesso facilitado à matériaprima e baixos custos de mão de obra.

Observou-se também que a DC estava migrando de uma EC que enfatizava a liderança em custos para uma em que a diferenciação (pela alta qualidade dos produtos) passava a ser priorizada, mas sem prejuízo de um controle rigoroso dos custos. E com este movimento, as estratégias de marketing e de produção requeridas tornavam-se mais complexas.

Destacaram-se como pontos fortes nos âmbitos da EC e 
da EP (esta será detalhada à frente): baixo custo de produção; alta qualidade de produção dos elementos principais dos produtos; pequena concorrência no mercado interno; acesso a matérias-primas; tecnologia atualizada de produção; imagem da empresa (corporação) no mercado interno; apoio da corporação à DC; espaço para crescimento; investimentos recentes da empresa na DC; consolidação da unidade de negócios; parcerias com fornecedores e clientes.

Como pontos que requerem aperfeiçoamentos, obteve-se: qualidade de acabamento dos produtos; prazos de entrega; serviços de marketing; imagem no mercado interno e externo; escala de produção; alguns conflitos com a corporação; dificuldades de ampliação da capacidade; dificuldades de manutenção / ampliação dos investimentos; consolidação da divisão no mercado interno e crescimento no mercado externo; inexistência de planejamento formalizado e sistemático; pouca abrangência dos sistemas de medidas de desempenho; excesso de refugo e retrabalho; gargalo no acabamento final( limpeza e montagem) e a dependência de esforço próprio para desenvolvimento de tecnologia de produtos.

A formulação e reformulação da EC da DC ocorria até recentemente na empresa em reuniões anuais, quando são definidos os planos de investimentos e as metas a serem atingidas pelas unidades de negócios. Agora a empresa realiza reuniões com essa pauta a cada três meses, implementando um sistema de formulação e avaliação de planos denominado "rolling forecast".

\subsection{Identificação da Estratégia de Produção da} Divisão Cosméticos

Para identificar e discutir a estratégia de produção da unidade de negócios foram utilizados os dados obtidos em entrevistas que contemplaram um levantamento das prioridades competitivas, dos programas e mudanças principais implementados nas áreas de decisão da produção.

Quanto às prioridade competitivas, após compilar e discutir as respostas dos dois entrevistados, concluiu-se que a melhoria da qualidade de acabamento (estética), a flexibilidade (de produto, de volume e de processo (mix de produção)) e a entrega são as principais preocupações da unidade. Em um segundo plano de preocupações está a manutenção dos baixos custos de produção.

A importância dessas dimensões competitivas pode ser explicada quando se analisa a EC da divisão, ou seja, uma estratégia que busca diferenciação para atender as expectativas de mercado quanto à qualidade, ao acabamento e aos prazos de entrega. Além disso, como a DC pretende desenvolver os mercados interno e externo, a produção sofre pressões maiores para a melhoria da qualidade, o aumento da flexibilidade e a redução de custos.

Analisando-se as questões relacionadas aos objetivos da produção, verifica-se que a ampliação da capacidade, a redução de retrabalho e refugo e o aumento da padronização de produtos e materiais são os principais objetivos da unidade. Através da ampliação da capacidade, a unidade espera resolver problemas quanto à flexibilidade do produto, flexibilidade de volume, flexibilidade de processo, qualidade e de entrega (reduzindo o impacto de alguns gargalos de produção). Com a redução de retrabalho e de refugo, poderá reduzir custos e melhorar a qualidade de conformação. $\mathrm{O}$ aumento da padronização de produtos e de materiais contribuirá para a redução de custos.

Além desses, a divisão destaca outros objetivos como importantes, tais como reduzir o tempo de produção (para melhorar entrega e custo), reduzir o ciclo de desenvolvimento de novos produtos (para melhorar custo e flexibilidade de produto), diminuir o tempo de produção (para melhorar custo e entrega), aumentar a quantidade de produtos produzidos (para aumentar a flexibilidade) e reduzir o tempo de troca de ferramentas (para melhorar custo e flexibilidade).

De acordo com a estratégia de produção, devem-se fazer algumas escolhas quanto aos programas ou planos de ação a serem implementados em cada área de decisão, de modo que os objetivos sejam alcançados.

Os dados obtidos quanto à implementação dos programas na DC são apresentados a seguir. 


\begin{tabular}{l|l}
\hline Áreas de programas & Programas implantados e funcionando \\
\hline Gestão & - Sistemas de informação na produção \\
& - Organização dos componentes, produtos e materiais em famílias \\
& - Codificação de materiais \\
& - Sistema ERP \\
\hline Organização & - Segurança do trabalho \\
& - Métodos de prevenção de acidentes \\
\hline Qualidade & - Inspeção de matéria-prima, de produto final e no processo \\
\hline Automação & - CAD \\
\hline Controle & - Controle de custos \\
\hline Manutenção & - Manutenção corretiva \\
\hline
\end{tabular}

\begin{tabular}{l|l}
\hline Áreas de programas & Programas parcialmente implantados \\
\hline Gestão & - JT \\
\hline Organização & - Treinamento de chefes e gerentes \\
\hline Tecnologia & - P\&D para introdução de novos produtos \\
\hline
\end{tabular}

\begin{tabular}{l|l}
\hline Áreas de programas & Programas que estão sendo estudados ou iniciando a implantação \\
\hline Gestão & - Reorganização da produção \\
& - ovos processos para novos produtos \\
& - Novos processos para velhos produtos \\
& - Restaurar planta física \\
& - Ligar a EP à EC \\
\hline Organização & - Times de trabalho interfuncionais \\
& - Avaliação do desempenho da MO \\
& - Ampliar o conjunto de tarefas da MO ou dar mais responsabilidade \\
& - Treinamento operacional da MO \\
& - Motivação de funcionários \\
& - Contratação de novas habilidades \\
\hline Tecnologia & - Capacitação tecnológica através de compra de tecnologia \\
& - Adaptação ou aperfeiçoamento de produtos, processos, equipamentos \\
e métodos
\end{tabular}


Pode-se destacar que os programas que estão implantados e funcionando na divisão são aqueles relacionados principalmente à gestão da produção e à organização. Os programas que estão parcialmente implantados são apenas três: um na área de gestão, um na área de organização e um na área de tecnologia. Já os programas que estão sendo estudados ou iniciando a implantação concentram-se principalmente nas áreas de organização, gestão e controle.

A empresa vinha até há alguns meses atrás priorizando a implementação de programas nas áreas infra- estruturais (gestão e organização).

Além desses programas, que constavam no roteiro de entrevistas, foram apontados pelas pessoas entrevistadas alguns programas que estão em andamento e cuja implantação é urgente. Estes programas, apresentados a seguir, mostram que realmente há forte preocupação na DC com a qualidade e os custos.

Observa-se, portanto, que os objetivos de melhoria da qualidade e de redução de custos vinham sendo sustentados por meio da implementação de técnicas organizacionais nas áreas infra-estruturais da produção.

\begin{tabular}{l|l}
\hline Áreas de programas & Programas que estão em andamento e são urgentes \\
\hline Gestão & - Padronização \\
& -Produtividade da estação de trabalho \\
& - Reestruturação da árvore dos produtos (tempos e quantidades) \\
& - Mudar layout \\
\hline Qualidade & - Implantação do CEP \\
& - Identificação e redução de refugos e retrabalhos \\
\hline
\end{tabular}

Em conjunto, todos esses programas nas áreas infraestruturais contribuem para melhorias em diversas dimensões competitivas, pois permitem aperfeiçoamentos em qualquer uma delas, através, principalmente, da capacitação e motivação da mão-de-obra direta e indireta.

A opção pela implementação de mudanças nas áreas infra-estruturais deve-se à difusão mais rápida e abrangente das técnicas organizacionais e de seu custo mais baixo, em relação às mudanças em produtos, processos, equipamentos e instalações.

Observa-se, no entanto, que os investimentos nas áreas estruturais que vinham sendo adiados pela divisão tornaram-se necessários e urgentes, principalmente, para melhoria da flexibilidade, da qualidade e das entregas. Estes investimentos estão sendo realizados na ampliação da capacidade através da aquisição de novas máquinas, da ampliação de espaço físico e do relayout da produção. A aquisição de novos equipamentos tem como objetivos principais, além do aumento da capacidade, a redução de gargalo, a redução de refugos e retrabalhos e a produção de novos produtos. No entanto, como há incerteza quanto aos resultados que poderão ser obtidos através da implantação dos novos equipamentos, parece que, pelo menos no curto prazo, , será difícil manter os custos nos patamares atuais.

Além desses investimentos, algumas mudanças foram consideradas como relevantes pela divisão como o controle rígido de materiais, a implantação de um sistema ERP, a ISO9003 e, num futuro próximo, a ISO9001, investimentos em treinamento e capacitação ("GMP" Good Manufacturing Practices) e aperfeiçoamentos das parcerias com clientes e fornecedores.

Estas mudanças são recursos empregados para obtenção de resultados nas várias dimensões competitivas, ou seja, seguem uma tendência de modernização, dando suporte e trazendo contribuições, em geral, para quaisquer 
PRODUÇÃO

das prioridades competitivas. Aparentemente, haverá ainda espaço (sem trade offs) para o desenvolvimento de programas nas áreas infra-estruturais que contribuam, simultaneamente, para algumas das prioridades competitivas da produção. A especificação de programas específicos direcionados para determinadas (e bem definidas) prioridades dependerá ainda da avaliação dos impactos (nas prioridades competitivas) das mudanças mencionadas nas áreas estruturais. Tal especificação será tão mais necessária quanto maior for o número de trade offs entre as dimensões então identificadas.

Os programas e mudanças implementados pela divisão parecem ser coerentes erıtre si, pois reforçam as mesmas competências da produção. E são também coerentes com as prioridades competitivas mencionadas como mais importantes: melhoria da qualidade, da flexibilidade, da entrega e manutenção dos baixos custos de produção.

Embora a divisão esteja investindo nas áreas estruturais, para aumentar sua competitividade e se consolidar no mercado, há a necessidade de continuar a empreender esforços nas áreas infra-estruturais, pois há ainda muito espaço a ser explorado.

Quanto à formulação da EP na DC, a equipe constatou que, embora haja um conhecimento já aprofundado do assunto, essa estratégia vem sendo formulada e reformulada de modo informal em reuniões que os gerentes e seus subordinados, uma equipe pequena, realizam semanalmente. O principal problema identificado quanto a essa sistemática é a não formalização - para comunicação com o restante das pessoas na DC e na empresa - e a dificuldade, em conseqüência, de se realizarem avaliações periódicas de modo aprofundado.

Aparentemente, não seria complicado se implantar um Sistema de Gestão Estratégica da Produção (SGEP) para avaliação e reformulação da estratégia de produção, pois a equipe de comando é relativamente pequena (três pessoas) e coesa. Um esforço maior seria necessário apenas para a concepção de tal sistema, identificando-se as medidas de desempenho (indicadores e índices) relacionadas às prioridades competitivas e aos programas implementados e definindo-se os procedimentos de obtenção e processamento dos dados e das informações. A implementação de tal sistema propiciaria a fundamentação necessária para a formulação de novos programas ou planos de ação.

\section{Considerações Finais}

Um importante aspecto a ser ressaltado neste trabalho é o resultado da experiência de realização desta pesquisa por uma equipe de profissionais e pesquisadores. Os profissionais que trabalham na divisão passaram a contar, no período de realização deste trabalho, com uma equipe de assessores especializados na área de EP. Como na empresa esta área era pouco desenvolvida, a realização do trabalho proporcionou também aos profissionais uma oportunidade de pensarem sobre o assunto, acumularem conhecimentos e participarem efetivamente do processo de desenvolvimento da EP e de, então, adquirirem condições de continuar a desenvolver a própria estratégia.

Já os pesquisadores puderam desenvolver melhor seu trabalho de pesquisa, a partir de um amplo conhecimento da realidade da empresa, e com a segurança de que as informações eram fidedignas. Além disso, puderam contar com os conhecimentos dos profissionais que vivenciam os problemas do dia-a-dia da produção.

Outro aspecto a ser destacado no trabalho realizado em equipe é que, como a divisão não tinha uma estratégia formalizada, ao mesmo tempo em que a estratégia era identificada, um novo processo de elaboração ia sendo criado. Desse modo, ganharam os profissionais, porque tiveram seu trabalho melhor delineado e também ganharam os pesquisadores ao participar diretamente da discussão e reelaboração da EP em um processo de pesquisa-ação.

Neste trabalho, embora a maior preocupação tenha sido com o conteúdo da EP, sua identificação e discussão, também trabalhou-se com o processo de formulação, sem entretanto um aprofundamento maior. Houve preocupação em se identificar e discutir o processo atual e indicar 
maneiras de aperfeiçoá-lo para torná-lo mais sistemático e técnico, utilizando-se métodos específicos para formulação como, por exemplo, a matriz QFD (Quality Function Deployment) e a matriz de importância-desempenho. No entanto, como este estudo ainda não foi finalizado, o processo de formulação e a proposição de um Sistema de Gestão Estratégica da Produção (SGEP) deverá ser objeto de um próximo artigo.

No processo de identificação e discussão, as estratégias competitiva e de produção foram reelaboradas, procurando-se torná-las mais claras e melhor integradas aos novos programas de ação, de modo que os resultados sejam alcançados mais rapidamente. Nesse processo, as seguintes sugestões foram dadas: criar e implementar um sistema de avaliação de desempenho; introduzir técnicas de motivação, treinamento e participação da mão de obra; melhorar a integração da DC com as funções de apoio da empresa (da corporação) e utilizar o controle do fluxo de caixa como meio para aperfeiçoar a tomada de decisões.

Além disso, a divisão deve voltar a despender esforços e delinear outros programas para melhorias nas áreas de decisão infra-estruturais, logo após os ajustes que se seguirão à etapa de implantação de novos equipamentos e de "relayout" (já programados). A hipótese de que as empresas concentram alternadamente mudanças nas áreas estruturais e nas áreas infra-estruturais foi reforçada pelas decisões tomadas na empresa. A sugestão neste caso é que a empresa procure desenvolvê-las e implementá-las de modo integrado e em paralelo (simultaneamente, se possível), o que poderia torná-las, em conjunto, mais eficazes e ainda reduzir o tempo total de sua implementação.

No geral, as estratégias são compatíveis, necessitando de pequenos ajustes. Mas há que se considerar a necessidade de detalhamento dos programas na área de produção e na área de marketing, para se buscar, de modo eficaz, o desenvolvimento dos mercados interno e externo. E há, ainda, a necessidade de adoção de um processo formal de formulação e de desdobramento e implementação das estratégias.

\section{Referências Bibliográficas}

PRODUÇÃO

COUTINHO, L.G.; FERRAZ, J.C. Estudo da competitividade da indústria brasileira. Campinas, Papirus, 1994.

GARVIN, D. A. Manufacturing Strategic Planning. California Management Review, summer, 1993.

HAYES, R. H. Strategic planning - forward in reverse? Harvard Business Review, nov-dec, 1985.

HILL, T. Manufacturing Strategy - The Strategic Management of the Manufacturing Function. Londres, Open University, 1993

HÖRTE, S. A. et al. Manufacturing strategies in Sweden. International Journal of Production Research, v.25, n. 11, 1987.

LEONG, G. K. et al. Research in the Process and Content of Manufacturing Strategy. OMEGA, v.18, n. 2, 109$122,1990$.

MILLS, J. et al. A framework for the design of manufacturing strategy process: A contingency approach. Int. Journal of Operations \& Production Management, vol.15, $\mathrm{n}^{\circ}$ 4, 1995.

MONTGOMERY, C.A.; PORTER, M.E. Estratégia: a busca da vantagem competitiva. Rio de Janeiro, Campus, 1998.

OHMAE, K. Voltando à estratégia. In: MONTGOMERY, C.A.; PORTER, M.E. Estratégia: a busca da vantagem competitiva. Rio de Janeiro, Campus, 1998.

PORTER, M.E. Competitive advantage. New York, The Free Press, 1985. 
PRODUÇÃO

PORTER, M.E. Competitive strategy. New York, The

Free Press, 1980.

PRAHALAD, C.K.; HAMEL, G. A competência essencial da corporação. In: MONTGOMERY, C.A.; PORTER, M.E. Estratégia: a busca da vantagem competitiva. Rio de Janeiro, Campus, 1998.

QUINN, J. B. Strategies for change. In: QUINN, J. B.; MINTZBERG, H.; JAMES, R. M. The strategy process: concepts, contexts, and cases. Englewood Cliffs : Prentice-Hall, 1988.

SKINNER, W. Manufacturing-Missing Link in Corporate Strategy. Harvard Business Review, may-jun, 1969.

SWAMIDAS et al. Manufacturing Strategy Enviromental Uncertainty and Performance: A Path Analytic Model, Management Science, Vol. 33, n. 4, 1987.

SLACK, N. Vantagem Competitiva em Manufatura:

Atingindo Competitividade nas Operações Industriais, Ed. Atlas, 93

VANALLE, R. M. Estratégia de produção e prioridades competitivas no setor de autopeças. São Carlos, EESC-USP, Tese de doutorado, 1995.

WHEELWRIGHT, S. C. Manufacturing strategy: defining the missing link. Strategic Management Journal, v. 5, 1984.

VOSS, C.A Manufacturing Strategy - Process and Content. Ed. Chapman \& Hall, London, 1992 